\title{
Simultaneous bilateral cataract extraction in the UK
}

\author{
S Beatty, R K Aggarwal, D B David, M Guarro, H Jones, J L Pearce
}

\begin{abstract}
Aims/Background-Simultaneous bilateral extracapsular cataract extraction (SBCE) is not routinely performed in the developed world. This study investigated the safety and efficacy of this procedure as performed in the UK.

Methods-A review of 319 consecutive patients (638 eyes) who underwent SBCE with posterior chamber intraocular lens implantation is presented.

Results-The incidence of intraoperative complications was $7 \cdot 7 \%$. These included posterior capsule rupture $(0.8 \%)$ and vitreous loss $(0 \cdot 3 \%)$. Postoperative complications were seen in $8 \cdot 7 \%$ of operated eyes and included iris prolapse $(0 \cdot 3 \%)$ and raised intraocular pressure (3.0\%). Endophthalmitis occurred in one eye $(0 \cdot 15 \%)$. There were no major bilateral complications, and, in particular, no cases of bilateral endophthalmitis. Final visual acuities compared favourably with reports for unilateral surgery. Eighty two per cent achieved a final visual acuity of $6 / 12$ or better, and $41 \%$ tested $6 / 6$ or better. Conclusion-This study illustrates that SBCE is not associated with an increased incidence of complications and that the visual results are good. Where indicated, and under strict surgical protocol, this procedure is safe.
\end{abstract}

(Br f Ophthalmol 1995; 79: 1111-1114)

Simultaneous bilateral cataract extraction (SBCE) is a controversial subject. It is not routine practice in the developed world and consequently the few reports in the literature are based on experiences where the operating conditions are not satisfactory. ${ }^{12}$ The reluctance of many ophthalmologists to carry out this procedure rests on the fear of a bilateral complication leading to blindness in both eyes. The improved safety and effectiveness of modern cataract surgery ${ }^{3} 4$ suggest that the reservations ophthalmic surgeons have regarding SBCE should be readdressed. This study reports the visual results and complications of a large number of patients undergoing bilateral cataract extraction during one operating session in the UK.

\section{Patients and methods}

This was a retrospective study of 319 patients (638 eyes) who underwent simultaneous bilateral extracapsular cataract extraction with posterior chamber intraocular lens implantation. All procedures were performed between 1985 and 1991 at the Alexandra Hospital, Redditch. The data collected for all patients undergoing this procedure can be divided into three groups.

(1) Demographic and preoperative: these include coexisting ocular pathology, best corrected visual acuity, and general health characteristics. Underlying medical conditions were categorised according to the risk classification system of the American Society' of Anesthesiology (ASA). ${ }^{5}$

(2) Peroperative: grade of operating surgeon and intraoperative complications (surgical miosis, bulging iris, iris trauma, posterior capsule rupture, vitreous loss, and 'other intraoperative complications').

(3) Postoperative: hyphaema, iris prolapse, wound leak, striate keratopathy, raised intraocular pressure (IOP $>22 \mathrm{~mm} \mathrm{Hg}$ ), chronic uveitis, posterior capsular thickening, clinically significant cystoid macular oedema, retinal detachment, and endophthalmitis.

Best corrected final visual acuity and length of follow up were also recorded.

The protocol for patient selection was established before the commencement of this study and included informed and valid consent comprising a frank discussion between surgeon and patient regarding the possible dangers of SBCE; the option of sequential unilateral operations open to all candidates; significant bilateral lens opacities, each eye fulfilling our criteria for unilateral cataract extraction; unsuitability for local anaesthesia and fitness for general anaesthesia (in no case was simultaneous bilateral cataract extraction performed under a regional block); proceeding to operate on the second eye only if the first was free of serious complications such as posterior capsule rupture with vitreous loss or expulsive haemorrhage. Exclusion criteria included predisposition to ocular infection (blepharitis, atopic eczema, trichiasis) and intraocular pathology that might prolong the procedure (posterior synechiae, pseudoexfoliation).

The risk of endophthalmitis was minimised by administration of subconjunctival antibiotics (cefuroxime) at induction of anaesthesia, followed by lid and conjunctival toilet with $5 \%$ aqueous povidine-iodine. The usual strict aseptic technique associated with intraocular surgery was observed. The procedures were treated as two separate operations under one anaesthetic. The surgeon, assistant, and instrument nurse rescrubbed and regowned before operating on the second eye. A separate irrigation system and theatre trolley were used for each eye. Postoperatively two bottles of topical steroid-antibiotic preparation (Betnesol-N, Evans Medical, Horsham, Sussex) were used, one for each eye.

Our results are analysed and 95\% confidence intervals calculated. 


\section{Results}

A total of 319 patients (638 eyes) were included in this study (mean age 77.8 (range 45-96) years; 225 were females $(70 \cdot 6 \%)$ and 94 were males $(29 \cdot 4 \%)$. The results of anaesthetic assessment and the general medical characteristics of the patients are given in

Table 1 General health and $A S A$ scores*

\begin{tabular}{lc}
\hline & Percentage of patients \\
\hline ASA class: & \\
1 & $14 \cdot 4$ \\
2 & $59 \cdot 2$ \\
3 & $24 \cdot 7$ \\
4 & $1 \cdot 7$ \\
5 & $0 \cdot 0$ \\
Systemic disease: & $8 \cdot 5$ \\
Diabetes mellitus & $32 \cdot 0$ \\
Hypertension & $30 \cdot 0$ \\
Cardiorespiratory disease & \\
\hline
\end{tabular}

«The American Society of Anesthesiology physical status scale grades preoperative risk factors as follows: class $1=$ healthy patient; class $2=$ mild systemic disease without functional limitation; class $3=$ severe systemic disease with definite functional limitation; class $\mathbf{4}=$ severe systemic disease that

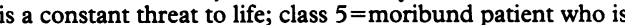
unlikely to survive 24 hours with or without operation.

Table 2 Pre-existing ophthalmic disease

\begin{tabular}{|c|c|c|c|c|c|c|}
\hline & \multicolumn{2}{|c|}{$\begin{array}{l}\text { Right eye } \\
(n=319)\end{array}$} & \multicolumn{2}{|c|}{$\begin{array}{l}\text { Left eye } \\
(n=319)\end{array}$} & \multicolumn{2}{|c|}{$\begin{array}{l}\text { Total eyes } \\
(n=638)\end{array}$} \\
\hline & $n$ & $\%$ & $n$ & $\%$ & $n$ & $\%$ \\
\hline $\begin{array}{l}\text { Glaucoma } \\
\text { ARMD } \\
\text { Diabetic retinopathy } \\
\text { Diabetic maculopathy }\end{array}$ & $\begin{array}{r}14 \\
33 \\
4 \\
3\end{array}$ & $\begin{array}{r}4 \cdot 3 \\
10 \cdot 3 \\
1 \cdot 2 \\
0 \cdot 9\end{array}$ & $\begin{array}{r}15 \\
35 \\
4 \\
3\end{array}$ & $\begin{array}{r}4 \cdot 7 \\
11 \cdot 0 \\
1 \cdot 2 \\
0.9\end{array}$ & $\begin{array}{r}29 \\
68 \\
8 \\
6\end{array}$ & $\begin{array}{r}4 \cdot 5 \\
10 \cdot 6 \\
1 \cdot 2 \\
0.9\end{array}$ \\
\hline
\end{tabular}

$\mathrm{ARMD}=$ age-related macular degeneration

Table 3 Intra-and postoperative complications among 319 patients (638 eyes) undergoing simultaneous bilateral cataract extraction. Confidence intervals apply only to patients because of non-independence of paired eyes

\begin{tabular}{|c|c|c|c|c|c|}
\hline \multirow[b]{2}{*}{ Complication type } & \multicolumn{2}{|c|}{ Eyes $(n=638)$} & \multicolumn{2}{|c|}{ Patients $(n=319)$} & \multirow{2}{*}{$\begin{array}{l}\text { Confidence } \\
\text { interval } \\
\%\end{array}$} \\
\hline & $n$ & $\%$ & $n$ & $\%$ & \\
\hline \multicolumn{6}{|l|}{ Intraoperative: } \\
\hline Surgical miosis & 30 & $4 \cdot 7$ & 20 & $6 \cdot 3$ & $3 \cdot 0,9 \cdot 0$ \\
\hline Bulging iris & 10 & $1 \cdot 6$ & 10 & $3 \cdot 2$ & $1 \cdot 5,6 \cdot 1$ \\
\hline Iris trauma & 2 & 0.3 & 2 & 0.6 & $0 \cdot 1,2 \cdot 2$ \\
\hline Posterior capsule rupture & 5 & 0.8 & 5 & 1.6 & $0.5,3.6$ \\
\hline \multirow{2}{*}{\multicolumn{6}{|c|}{ Postoperative: }} \\
\hline & & & & & \\
\hline Hyphaema & 5 & 0.8 & 4 & $1 \cdot 3$ & $0 \cdot 3,3 \cdot 3$ \\
\hline Striate keratopathy & 6 & 0.9 & 4 & 1.9 & $0 \cdot 7,4 \cdot 1$ \\
\hline Wound leak & 2 & 0.3 & 2 & 0.6 & $0 \cdot 1,2 \cdot 2$ \\
\hline Iris prolapse & 2 & 0.3 & 2 & 0.6 & $0 \cdot 1,2 \cdot 2$ \\
\hline IOP $>22 \mathrm{~mm} \mathrm{Hg}$ & 19 & 3.0 & 17 & $5 \cdot 3$ & $3 \cdot 1,8 \cdot 1$ \\
\hline Uveitis & 9 & 1.4 & 8 & 2.5 & $1 \cdot 1,5 \cdot 1$ \\
\hline CCMO & 12 & 1.9 & 12 & $3 \cdot 8$ & $1.9,6.4$ \\
\hline Endophthalmitis & 1 & $0 \cdot 15$ & 1 & 0.3 & $0.01,1.7$ \\
\hline
\end{tabular}

$\mathrm{IOP}=$ intraocular pressure. $\mathrm{CCMO}=$ clinically apparent cystoid macular oedema.

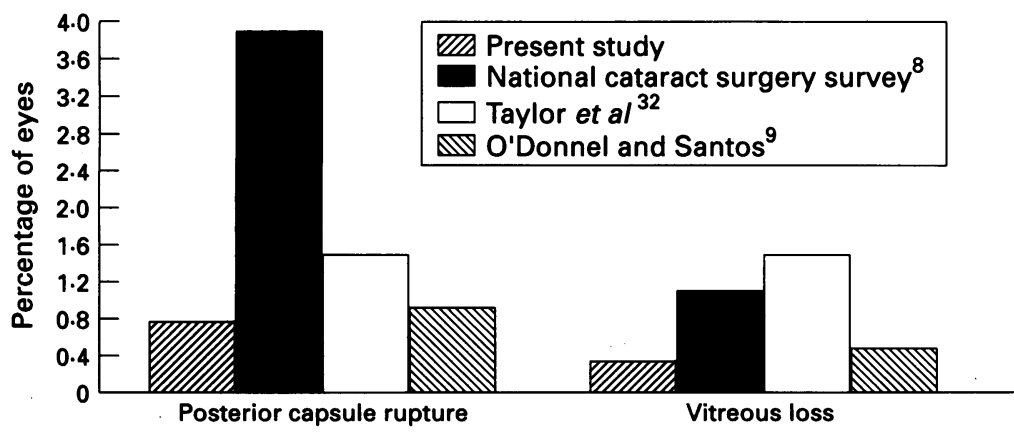

Figure 1 Comparison of intraoperative complication rate (\%) for simultaneous bilateral cataract extraction with previous reports of unilateral extracapsular cataract extraction.
Table 1 . Almost all $(98 \cdot 3 \%)$ were ascribed an ASA grade of 1,2 , or 3 . Pre-existing ocular comorbidity was seen in 111 eyes $(17 \cdot 4 \%)$ and included glaucoma, age-related macular degeneration, diabetic retinopathy, and diabetic maculopathy (Table 2).

One consultant (JLP) performed 613 $(96 \cdot 1 \%)$ of the cataract operations. A registrar operated on the second eye of the remaining 25 patients $(3.9 \%)$. In no case did a serious complication arising in the first eye necessitate cancellation of operation on the second eye.

Minor intraoperative complications included surgical miosis (30 eyes), bulging iris (10 eyes), and iris trauma (two eyes). The more serious complications were posterior capsule rupture (five eyes) and vitreous loss (two eyes) (Table 3; Fig 1). A posterior chamber intraocular lens was inserted in all cases.

Postoperatively the following complications were seen: hyphaema (five eyes); striate keratopathy (six eyes); wound leak (two eyes); iris prolapse (two eyes); postoperative rise in intraocular pressure (19 eyes); chronic uveitis (nine eyes); clinically apparent cystoid macular oedema (CCMO) (12 eyes) (Table 3; Fig 2). There were no cases of postoperative retinal detachment. Endophthalmitis occurred in one eye $(0 \cdot 15 \%)$ (Fig 2$)$.

Bilateral complications occurred in six patients $(1 \cdot 8 \%)$ and included hyphaema, striate keratopathy, raised intraocular pressure, and chronic uveitis (Table 4). These 12 eyes all achieved a final visual acuity of $6 / 12$ or better.

Posterior capsular thickening was seen in 83 eyes $(13 \%)$. The mean follow up time was $49 \cdot 15$ weeks (range 3 to 460 weeks; median 26 weeks).

A best corrected final visual acuity of $6 / 12$ or better was seen in 514 eyes $(82 \cdot 1 \%)$. Forty one per cent tested $6 / 6$ or better (Fig 3 ). All patients with a best corrected final visual acuity of $6 / 36$ or worse had pre-existing ophthalmic pathology to account for the poor result.

\section{Discussion}

In the May 1988 issue of the fournal of Cataract and Refractive Surgery, implant surgeons around the world were posed the question 'Are there any indications for simultaneous bilateral cataract and intraocular lens surgery?'. 6 The response strongly indicated that although some surgeons had performed this procedure, the vast majority were opposed to the concept. Similarly, in the recent guidelines for cataract surgery published by the Royal College of Ophthalmologists bilateral cataract surgery at one sitting is not advised except in exceptional circumstances. ${ }^{7}$

In this study peroperative complications were seen in $7.7 \%$ of operated eyes. The incidence of posterior capsule rupture was $0.8 \%$ and this compares favourably with reports in the literature $(0 \%$ to $8 \%)$ for unilateral extracapsular cataract extraction (ECCE).$^{8-17}$ Vitreous was lost in $0.3 \%$ of eyes, a similar rate to some studies for unilateral surgery $(0.09 \% \text { to } 0.46 \%)^{9111417}$ and much lower than others $(1 \cdot 1 \%$ to $3 \cdot 3 \%) .81215$ Iris 


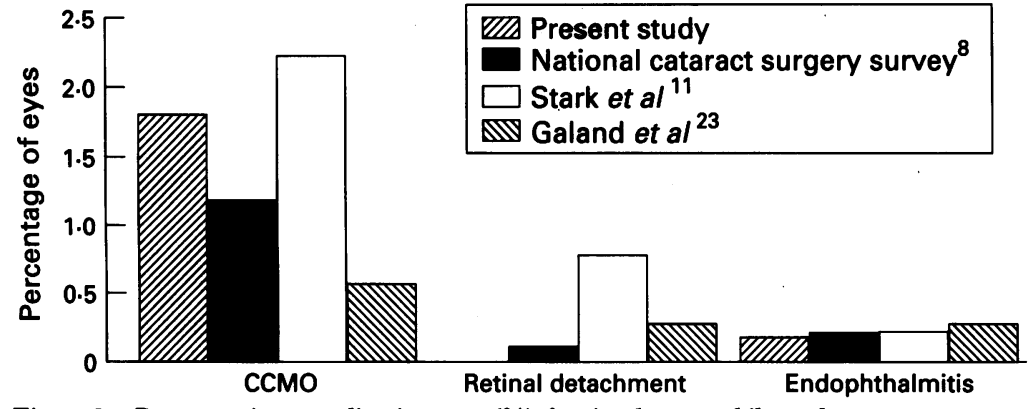

Figure 2 Postoperative complication rate (\%) for simultaneous bilateral cataract extraction and previous reports of unilateral extracapsular cataract extraction (CCMO = clinically apparent cystoid macular oedema).

trauma was encountered in $0.3 \%$ of eyes, less than previously published data $(9 \cdot 1 \%$, $3.5 \%) .1619$ There were no reports in the literature with which to compare our incidence of surgical miosis $(4 \cdot 7 \%)$ and bulging iris $(1 \cdot 6 \%)$. This low rate of intraoperative complications probably reflects the experience of the surgeon performing the vast majority of operations.

The incidence of postoperative hyphaema, iris prolapse, uveitis, and CCMO in this study compares favourably with their respective pooled postoperative complication rates for ECCE. ${ }^{3} \mathrm{~A}$ postoperative rise in intraocular pressure was seen in $3.0 \%$ of eyes, again consistent with previous reports. ${ }^{12} 15$

Our incidence of posterior capsular thickening was $13 \%$, in keeping with reports in the literature $(10.6 \%$ to $35 \%)$ where duration of follow up was comparable. ${ }^{1220-22}$

We believe that the reluctance of many ophthalmic surgeons to perform simultaneous intraocular surgery on both eyes is based on the fear of bilateral endophthalmitis. In this series of 638 cataract extractions, one eye developed this complication, representing an incidence of $0.15 \%$. The patient presented on the eighth postoperative day complaining of pain and reduced vision in the right eye. Vitreous samples grew Streptococcus faecalis. The condition did not respond to intravitreal, topical, and systemic antibiotics and the eye eventually required enucleation. Measures taken to avoid cross infection to the other eye included intravenous antibiotics; frequent topical antibiotics instilled into both eyes (left eye first); separate bottles of eyedrops for each

Table 4 Bilateral postoperative complications

\begin{tabular}{lll}
\hline Complication type & $n$ & Incidence (\%) \\
\hline Hyphaema & 1 & 0.03 \\
Striate keratopathy & 2 & 0.06 \\
IOP >22 mm Hg & 2 & 0.06 \\
Uveitis & 1 & 0.03 \\
\hline
\end{tabular}

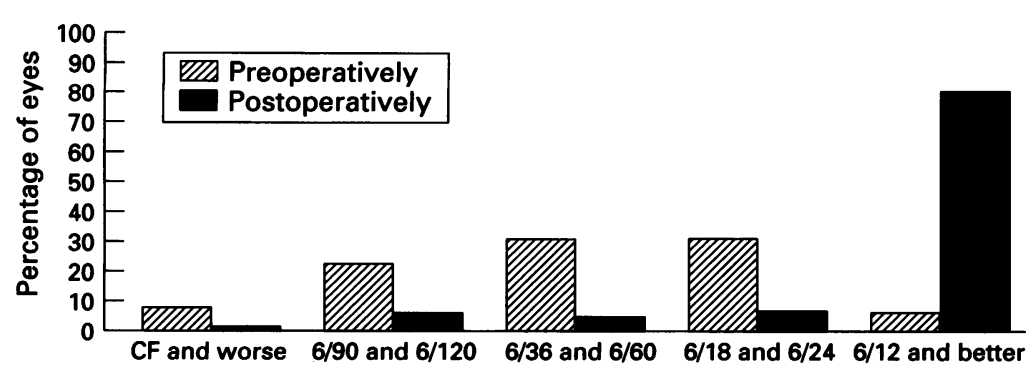

Figure 3 Distribution of visual acuities among eyes preoperatively and postoperatively. side; vigorous hand washing by nursing staff before and after administering topical medication in each eye; patient education as to the dangers of hand to face contact. The fellow eye was not affected and achieved a final visual acuity of 6/9. There were no cases of bilateral endophthalmitis. Previous studies reporting the incidence of endophthalmitis following unilateral ECCE vary from $0 \%$ to $1 \cdot 89 \% 8112023-27$ with a pooled rate of $0.35 \%{ }^{3}$

A final visual acuity of $6 / 12$ or better was achieved in $82 \%$ of the eyes in this study. This result is similar to that of other studies investigating visual outcome of unilateral extracapsular cataract extraction by experienced surgeons. ${ }^{28} 29$

The advantages of simultaneous bilateral cataract extraction can be divided into three categories - medical, social, and economic. The medical comprise a reduction in morbidity and mortality associated with avoiding an additional general anaesthetic in patients where this is the preferred form of anaesthesia; improved stereopsis, binocular visual acuity, and contrast sensitivity thresholds, and less glare disability, in the postoperative period compared with patients awaiting cataract extraction in the second eye. ${ }^{30}$ These factors, in theory, could contribute to a decrease in the number of accidents experienced by patients postoperatively. ${ }^{31}$ Also, the possibility that the patient with bilateral lens opacities undergoing unilateral surgery will not seek surgical attention for the other eye is avoided. The social benefits include a single admission to hospital, a reduction in the number of outpatient visits, and a shorter period of convalescence. The economic gains include savings resulting from one hospitalisation and a single general anaesthetic in patients unsuitable for local anaesthesia. In addition, although operating time would not be reduced, theatre time would be saved because of a single induction of anaesthesia. Other sources of financial savings would result from a shorter period of outpatient follow up and the need to dispense only one pair of glasses postoperatively. The patient would also benefit in this regard by requiring less time off work and fewer transport requirements to and from the hospital. However, the advantages of bilateral cataract extraction at one sitting should not be confused with the indications for performing this procedure.

In order to keep complications to a minimum simultaneous bilateral cataract surgery should only be performed by experienced surgeons in modern operating theatres. Patient selection is critical to the success of the procedure, and the protocol outlined above should be strictly observed.

We do not advocate routine SBCE. It should be considered where a general anaesthetic is to be used, especially in cases such as dense bilateral cataracts in the presence of known age-related macular degeneration or optic atrophy where it is impossible to predict the visual prognosis, and when the health of the patient is such that only one operative procedure is advisable. ${ }^{7}$ This study, the largest series reported in the UK, does demonstrate, however, the safety and efficacy 
of bilateral cataract surgery at one sitting and it may be appropriate to cautiously widen our criteria for performing this procedure. It remains a useful option for the ophthalmic surgeon.

1 Benezra D, Chirambo MC. Bilateral versus unilatera cataract extraction: advantages and complications. $\mathrm{Br}$ Ophthalmol 1978; 62: 770-3.

2 Joseph N, David R. Bilateral cataract extraction in one session: report on five years' experience. $B r \mathcal{F}$ Ophthalmo 1977; 61: 619-21.

3 Powe NR, Schein OD, Gieser SC, Tielsch JM, Luthra R, Javitt J, et al. Synthesis of the literature of visual acuity and complications following cataract extraction with intraocular lens implantation. Arch Ophthalmol 1994; 112: 239-52.

4 Acheson JF, McHugh JD, Falcon MG. Changing patterns of early complications in cataract surgery with new techniques: a surgical audit. $B r \mathcal{F}$ Ophthalmol 1988; 72 $481-4$.

5 American Society of Anaesthesiology: new classification of physical status. Anaesthesiology 1963; 24: 111 .

6 Anon. Are there any indications for simultaneous bilatera and intraocular lens surgery? F Cataract Refract Surg 1988, 14: 339-45.

7 The Royal College of Ophthalmologists. Guidelines for cataract surgery. London: RCO, 1995.

8 Desai P. The national cataract surgery survey: II Clinical outcomes. Eye 1993; 7: 489-94.

9 O'Donnell Jr FE, Santos BA. Prospective study of posterior capsule-zonular disruption. $\mathcal{F}$ Cataract Refract Surg 1990; 16: $329-32$.

10 Simcoe CW. Simcoe posterior chamber lens: theory, techniques and results. $\mathcal{F} \mathrm{Am}$ Intraocul Implant Soc 1981; 7 154-7.

11 Stark WJ, Maumanee A, Datiles M. Intraocular lenses: complications and visual results. Trans Am Ophthalmol Soc 1983; 81: 280-309.

12 Barrett GD, Beasley H, Lorenzetti OJ, Rosenthal A. Multicenter trial of an intraocular hydrogel lens implant. Therect

13 David R, Tessler Z, Yagev R. Persistently raised intraocular pressures following extracapsular cataract extraction. $\mathrm{Br}$ pressures following extracapsula

14 McCaffrey M, Lusby FA. A review of $5843 \mathrm{M}$ style 30 intraocular lens implants. $\mathcal{F}$ Cataract Refract Surg 1986; 12: $278-80$

15 Kooner KS, Dulaney DD, Zimmerman TJ. Intraocular pressure following extracapsular cataract extraction and posterior chamber intraocular lens implantation. Ophthalmic Surg 1988; 19: 471-4.

16 Naeser K, Rask KL, Hansen TE. Morphological changes after extracapsular cataract extraction with implantation of posterior chamber lenses: a prospective clinical study. Acta Ophthalmol (Copenhagen) 1986; 64: 323-9.

17 Silvestri G, Shepherd WF, Johnston PB. Pseudophakic retinal detachment and postoperative visual acuity. Int Ophthalmol 1989; 15: 9-12.

19 Straatsma BR, Meyer KT, Bastek JV, Lightfoot DO. Posterior chamber intraocular lens implantation by ophthalmology residents. Ophthalmology 1983; 90: 27-35.

20 Allen AW, Zhang HR. Extracapsular cataract extraction: prognosis and complications with and without posteriorchamber intraocular lens implantations. Ann Ophthalmol 1987; 19: 329-33.

21 Cheng $\mathrm{H}$. Current status of cataract surgery: a review of personal experience. Eye 1987; 1: 551-6.

22 Azen SP, Hurt A, Steel D, Wilson J, Reinig J, Bernstein J, et al. Effects of the Shearing posterior chamber lens implants on the corneal endothelium. Am $\mathcal{f}$ Ophthalmol 1983; 95: 798-802.

23 Galand A, Van Oye R, Budo C, Goes F. Results of implantation in the capsular bag: a short term review of 1588 . Trans Ophthalmol Soc UK 1985; 104: 563-6.

24 Van Oye R, Budo C, Galand A, Foets B, Goes F. Two-year postoperative results of Galand lens implantation. 7 Cataract Refract Surg 1986; 12: 135-9.

25 Noble BA, Hayward JM, Huber C. Secondary evaluation of posterior capsule-zonular disruption. $\mathcal{f}$ Cataract Refract Surg 1990; 16: 329-32.

26 Barret GD, Constable IJ, Steward AD. Clinical results of hydrogel lens implantation. $f$ Cataract Refract Surg 1986; 12: 623-31.

27 Javitt JC, Vitale S, Canner JK, Street BA, Krakauer H, McBean AM, et al. National outcomes of cataract extraction. Arch Ophthalmol 1991; 109: 1085-9.

28 Kratz RP, Mazzocco TR, Davidson B, Colvard DM. The Shearing intraocular: a report of 1000 cases. Am Intraocul Implant Soc f 1981; 7: 55-7.

29 Stark WJ, Maumanee AE, Dangel ME, Martin NF, Hirst LW. Intraocular lenses. Experience at the Wilmer Institute. Ophthalmology 1982; 89: 104-8.

30 Laidlaw A, Harrad R. Can second eye cataract extraction be justified? Eye 1993; 7: 690-6.

31 Felson DT Anderson JJ, Hannan MT, Milton RC, Wilson PW, Kiel DP. Impaired vision and hip fracture. The Framingham Study. $f$ Am Geriatr Soc 1989; 37: 495-500

32 Taylor DM, Sachs SW, Stern AL. Aphakic cystoid macular oedema: longterm clinical observations. Surv Ophthalmol $1984 ; 28: 437-41$. 